\title{
Evaluación del comportamiento de la hemorragia uterina disfuncional en pacientes de dos instituciones de salud de Bogotá. ISS/IMI
}

\author{
William Onatra H., Jacinto Sánchez A. ${ }^{\text {; }}$ Ricardo Alvarado P. ${ }^{2}$; Gonzalo Vargas P. ${ }^{3}$; Gabriel Tovar R. ${ }^{4}$;
}

RESUMEN OBJETIVO: Conocer si el comportamiento del HUD en el ISS guarda la misma relación encontrada en IMI de la ciudad de Bogotá.

MATERIAL Y METODOS: Se analizaron 158 biopsias endometriales del IMI de Bogotá y se compararon con 474 del ISS-Clínica San Pedro Claver de Bogotá. Se clasificaron como Endometrio Funcional al secretor proliferativo y atrófico, como orgánicos: Endometritis, R. Ovulares, Hiperplasia simple o Atípica, Cervicitis, Pólipo. Como prueba estadística se aplicó Chi-cuadrado y diferencia de promedio y proporciones.

RESULTADOS: Al clasificar la HUD por grupos de edad, el comportamiento es similar en ambas instituciones tanto para la HUD (IMI 72\%, ISS 70\%) como para HUO (IMI 28\%, ISS 30\%) Al examinar los diferentes endometrios en la HUD se encuentra una diferencia significativa del E. secretor según edad (IMI 37\%, ISS 47\%) P<0.01 y el E. Proliferativo (IMI 58\%, ISS 21.9\%) P < 0.01 . Atribuible este hallazgo al mejor estado nutricional de la población estudiada o a la ingesta hormonal exógena.

CONCLUSION: En el diagnóstico clínico de HUD al comparar con la biopsia endometrial el $30 \%$ obedece a origen orgánico y se confirman los hallazgos de la literatura donde a medida que aumenta la edad, el endometrio se hace proliferativo y tiende a la hiperplasia.

PALABRAS CLAVES: Hemorragia Uterina Disfuncional, Hemorragia Uterina Orgánica, Endometrio Secretor Proliferativo, Hiperplasia Endometial.

SUMMARY OBJECTIVE: To know if the DUB behaviour in ISS keeps the same relation founded in IMI in Bogotá city.

MATERIAL AND METHODS: 158 endometrial biopsy of the IMI in Bogotá and have been analized and compared with the 474 of the ISS. San Pedro Claver Clinic in Bogotá and have been classified as functional endometrium secretory proliferative, as organic: endometritis, simple or typical hyperplasia, cervicitis, polyps. The square-chi statistic proof was applied and difference of average and proportion.

RESULTS: When the DUB is classified by age grops, the behaviour is similar in both institutions as much as for HUD (28\% IMI/30\% ISS). When the different endometrium were analyzed, is found in the HUD a significant differente of the secretor endometrium depending on age (37\% IMI / 47\% ISS) $\mathrm{P}<0.01$ and of the E. proliferative (58\% IMI / 21.9\% ISS) $\mathrm{P}<00.1$. These findings are due to a better nutritional condition or to the "Foreing hormonal diet"

CONCLUSION: In the DUB clinic diagnostic, when it is compared with the endometrium biopsy, the $30 \%$ obey to organic and are confirmed the literature findings that when the age increases the endometrium becomes prolific and tends to hyperplasia.

KEY WORDS: Dysfunctional Uterine Bleeding, Organic Uterine Bleeding Secretory, Proliferative and Hyperplasia.

\section{Introducción}

La Hemorragia Uterina Disfuncional (HUD) es un transtorno del ciclo menstrual; no es originada por lesión orgánica y se considera como un diagnóstico de exclusión (1). Compromete a la mujer desde la adolescencia hasta el climaterio. Se considera que la base del problema es debido a un transtorno del eje Hipotamo Hipófisis ovario con repercusión endometrial y su etiología es diferente según la edad de aparición. En la adolescencia los estudios de Knobil (2) demostraron que la maduración folicular durante la menarquía depende de la pulsatilidad de la

\footnotetext{
' Profesor Asistente. Departamento de Obstetricia y Ginecología. Facultad de Medicina, Universidad Nacional de Colombia. Unidad de Endocrinología Ginecológica. Instituto Materno Infantil. Bogotá.

2 Profesor Asociado. Departamento de Patología. Facultad de Medicina. Universidad Nacional de Colombia. Instituto Materno Infantil. Bogotá.

${ }^{3}$ Estadístico. MSP. Profesor Asociado. Departamento de Medicina Preventiva. Facultad de Medicina. Universidad Nacional de Colombia.

${ }^{4}$ Especialista en Obstetricia y Ginecología. Instituto de Seguros Sociales. Clínica San Pedro Claver. Bogotá.
}

GnRH y que la alteración de sus pulsos determinan un pobre desarrollo folicular y un cuerpo lúteo insuficiente. En la vida reproductiva puede ser de origen ovulatorio como en la insuficiencia de cuerpo lúteo o anovulatorio por alteración en el mecanismo de retroalimentación. Durante la pre y post menopausia el defecto de receptores a nivel del ovario y la nula respuesta a las gonadotropinas es la causa más aceptada (3).

Es necesario iniciar el estudio descartando factores orgánicos tales como transtornos de la coagulación, hiper o hipotiroidismo, pólipos, miomas uterinos, lesiones vulvovaginales, cervicales y la patología del embarazo (4).

La HUD es responsable de un 10 a 35\% de la patología ginecológica y de un 25-30\% de las intervenciones quirúrgicas (5). En la adolescencia afecta entre el 4 y $20 \%$, en la vida reproductiva entre el $30-40 \%$ y en la menopausia hasta el $50 \%$ (6). En un estudio retrospectivo realizado por Beazley en Londres sobre un total de 287 casos de histerectomía la HUD fue en un $60 \%$ funcional, $31 \%$ orgánica y $7 \%$ mixta (7). En nuestro medio en Antioquia se dan cifras 69/31\% (8) y en IMI de Bogotá sobre 158 biopsias endometriales se 
obtiene $72 \%$ funcional y $28 \%$ orgánica (9). Fue interés de los autores comparar este último trabajo con el de una entidad de seguridad social de características demográficas diferentes.

\section{Material y métodos}

Se realiza un estudio comparativo retrospectivo tomando los resultados de patología en dos instituciones de salud como el Instituto Materno Infantil de clase socioeconómica baja y la Clínica San Pedro Claver de Bogotá de la clase económica media. La primera muestra incluye 158 de 300 casos atendidos durante 6 meses (1987-88) en IMI y la segunda 474 casos en el ISS en 12 meses (1990). Todas consultaron en el servicio de urgencias por hemorragia uterina clasificándolas como HUD, se les practicó legradobiopsia. En los resultados de patología se optó la siguiente clasificación: endometrio funcional, el secretor proliferativo, atrófico e hipersecretor, y como endometrio con lesión orgánica la endometritis, restos ovulares, cervicitis, hiperplasia endometrial, carcinoma endometrial, pólipos.

Las pacientes se clasificaron según la edad en 3 grandes grupos $<20$ años, 20-40 y 40-60 años. Para la conclusión de resultados válidos se aplicó como prueba estadística chicuadrado, la prueba de proporciones y promedios utilizadas en estos casos.

\section{Resultados}

El comportamiento etiológico de la HUD en ambas instituciones es comparable no encontrando diferencias significativas entre la HUD y la HUO. Tabla 1 . Al clasificar por grupos etarios Tabla 2 tampoco hay diferencias en las dos instituciones a excepción del grupo entre 30-39 años donde la hemorragia de origen orgánico es mayor en ISS que en el IMI $(\mathrm{P}<0.1)$.

Tabla 1

ETIOLOGIA DE LA HUD: IMI/ISS

\begin{tabular}{|lrrrrl|}
\hline Tipo de & \multicolumn{1}{c}{ IMI } & \multicolumn{1}{l|}{ ISS } & P.Signif. \\
Hemorragia & n & \multicolumn{1}{c|}{$\%$} & \multicolumn{1}{c|}{$\mathbf{n}$} & $\boldsymbol{\%}$ & X2 \\
\hline Disfuncional & 114 & 72 & 332 & 70 & NS \\
Ogánica & 44 & 28 & 142 & 30 & NS \\
Total & 158 & 100 & 474 & 100 & \\
\hline
\end{tabular}

PS: Prueba de significancia.

Tabla 2

GRUPOS DE EDAD/TIPO DE ENDOMETRIO

EN LA HUD

\begin{tabular}{|c|c|c|c|c|c|c|c|c|c|c|}
\hline \multirow{2}{*}{\multicolumn{2}{|c|}{$\begin{array}{l}\text { Grupos de } \\
\text { Edad/Años }\end{array}$}} & \multirow{3}{*}{$\begin{array}{r}\text { IMI } \\
\%\end{array}$} & \multicolumn{3}{|c|}{ HUD } & \multicolumn{5}{|c|}{ HUO } \\
\hline & & & \multicolumn{2}{|c|}{ ISS } & \multirow{2}{*}{$\begin{array}{l}\text { PS } \\
\mathrm{X} 2 \\
\end{array}$} & \multicolumn{2}{|r|}{ IMI } & \multicolumn{2}{|r|}{ ISS } & \multirow{2}{*}{$\begin{array}{r}\text { PS } \\
\times 2 \\
\end{array}$} \\
\hline & n & & n & $\%$ & & $n$ & $\%$ & $\mathrm{n}$ & $\%$ & \\
\hline $15-19$ & 4 & 2.5 & 4 & 0.8 & NS & 1 & 0.6 & & & \\
\hline 2029 & 32 & 20.2 & 86 & 18.2 & NS & 14 & 8.9 & 36 & 7.6 & NS \\
\hline 3039 & 31 & 19.6 & 113 & 23.8 & NS & 5 & 3.2 & 49 & 10.3 & $\mathrm{P}<0$. \\
\hline 4049 & 37 & 23.4 & 111 & 23.4 & $=$ & 18 & 11.4 & 46 & 9.7 & NS \\
\hline 5059 & 10 & 6.3 & 18 & 3.8 & NS & 6 & 3.8 & 11 & 2.3 & NS \\
\hline Total & 114 & 72.0 & 332 & 70.0 & & 44 & 28.0 & 142 & 30.0 & \\
\hline
\end{tabular}

En la tabla 3 se analiza el tipo de Endometrio Funcional por grupo de edad, encontrándose un predominio del Endometrio Secretor por encima de los 20 años en el ISS, frente al IMI. Por el contrario en el IMI hay predominio delE. Proliferativo y atrófico con diferencias significativas $(P<0.01)$.

Tabla 3

GRUPOS DE EDAD/TIPO DE ENDOMETRIO EN LA H.U. DISFUNCIONAL

\begin{tabular}{|c|c|c|c|c|c|c|c|c|c|c|c|c|c|c|c|}
\hline \multicolumn{16}{|c|}{ Hemorragia Uterina Disfuncional } \\
\hline \multirow{3}{*}{$\begin{array}{c}\text { Grupo de } \\
\text { Edad/años }\end{array}$} & \multicolumn{4}{|c|}{ E. Secretor } & \multirow{3}{*}{$\begin{array}{l}\text { PS } \\
X_{2}\end{array}$} & \multicolumn{4}{|c|}{ E. Proliferativo } & \multirow{3}{*}{$\begin{array}{l}\text { PS } \\
\mathrm{X} 2\end{array}$} & \multicolumn{4}{|c|}{ E. Atrófico } & \multirow{3}{*}{$\begin{array}{l}\text { PS } \\
\mathrm{X} 2\end{array}$} \\
\hline & \multicolumn{2}{|c|}{ IMI } & \multicolumn{2}{|c|}{ ISS } & & \multicolumn{2}{|c|}{ IMI } & \multicolumn{2}{|c|}{ ISS } & & \multicolumn{2}{|c|}{ IMI } & \multicolumn{2}{|c|}{ ISS } & \\
\hline & $\mathbf{n}$ & $\%$ & $\mathbf{n}$ & $\%$ & & $\mathbf{n}$ & $\%$ & $\mathbf{n}$ & $\%$ & & $\mathbf{n}$ & $\%$ & $\mathbf{n}$ & $\%$ & \\
\hline $20<$ & 2 & 1.3 & 2 & 0.42 & NS & 2 & 1.3 & 2 & 0.42 & NS & & & & & \\
\hline 2039 & 27 & 17.1 & 144 & 30.3 & $P<0.001$ & 33 & 20.8 & 52 & 10.9 & $\mathrm{P}<0.001$ & 2 & 1.3 & 3 & 0.63 & $P<0.001$ \\
\hline$>40$ & 14 & 8.8 & 77 & 16.2 & $\mathrm{P}<0.001$ & 32 & 20.2 & 50 & 10.5 & $P<0.001$ & 2 & 1.3 & 2 & 0.42 & $\mathrm{P}<0.001$ \\
\hline Total & 47 & 29.7 & 223 & 47.0 & 67 & 42.4 & 104 & 21.9 & & & 4 & 2.5 & 5 & 1.00 & \\
\hline
\end{tabular}

Las tablas 4A Y 4B compara la patología orgánica en las dos instituciones por grupos de edad. Se aprecia que el comportamiento en general de causa orgánica es similar en ambas instituciones donde la primera causa es la endometritis y donde el Endometrio estimulado por hormonas exógenas predomina en el ISS. Es llamativo que un grupo de edad entre 20-40 años los restos ovulares y la hiperplasia predomine en ISS, frente al IMI $(\mathrm{P}<0.05)$. La cervicitis, pólipo y CA. Endometrial no mostró diferencias en las dos instituciones.

\section{Discusión}

Las alteraciones del ciclo menstrual son una frecuente causa de consulta en la práctica ginecológica. La frecuencia de la HUD oscila entre $10-35 \%$ y un $20-25 \%$ de todas las operaciones ginecológicas $(5,9)$. Al clasificarla por grupos de edad adolescencia ( $<20$ años), vida reproductiva (20-30 años), menopausia (40-60 años $>$ ) la literatura informa $20-30$ y $50 \%$ respectivamente mientras en un estudio previo en el IMI de Bogotá se obtienen cifras de 455 y $41 \%(6,9-10)$. 
Tabla 4A

GRUPOS DE EDAD/TIPO DE ENDOMETRIO EN LA H.U. ORGANICA

\begin{tabular}{|c|c|c|c|c|c|c|c|c|c|c|c|c|c|c|c|c|c|c|c|c|}
\hline \multirow{4}{*}{$\begin{array}{l}\text { Grupo de } \\
\text { Edad/Años }\end{array}$} & \multicolumn{19}{|c|}{ HEMORRAGIA UTERINA ORGANICA } & \multirow{4}{*}{ PS } \\
\hline & \multicolumn{4}{|c|}{ Endometritis } & \multirow[t]{3}{*}{ PS } & \multicolumn{4}{|c|}{ R.Ovulares } & \multirow[t]{3}{*}{ PS } & \multicolumn{4}{|c|}{ Hiperplasia E. } & \multirow[t]{3}{*}{ PS } & \multicolumn{4}{|c|}{ Cervicitis } & \\
\hline & \multicolumn{2}{|c|}{ IMI } & \multicolumn{2}{|c|}{ ISS } & & \multicolumn{2}{|c|}{ IMI } & \multicolumn{2}{|c|}{ ISS } & & \multicolumn{2}{|c|}{ IMI } & \multicolumn{2}{|c|}{ ISS } & & \multicolumn{2}{|c|}{ IMI } & \multicolumn{2}{|c|}{ ISS } & \\
\hline & $\mathbf{n}$ & $\%$ & $\mathbf{n}$ & $\%$ & & $\mathbf{n}$ & $\%$ & $\mathbf{n}$ & $\%$ & & $\mathbf{n}$ & $\%$ & $\mathbf{n}$ & $\%$ & & $\mathbf{n}$ & 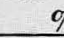 & & $\%$ & \\
\hline $20<$ & 1 & 0.6 & & & & & & & & & & & & & & & & & & \\
\hline 2039 & 10 & 6.3 & 27 & 5.7 & NS & 6 & 3.8 & 31 & 6.5 & $\mathrm{P}<0.05$ & 1 & 0.6 & 14 & 2.9 & $P<0.05$ & 1 & 0. & 3 & 0.6 & $=$ \\
\hline 4059 & 5 & 3.1 & 14 & 2.9 & NS & 1 & 0.6 & 11 & 2.3 & NS & 12 & 7.6 & 19 & 4.0 & NS & 2 & 1. & 2 & 0.4 & NS \\
\hline Total & 16 & 10.1 & 41 & 8.6 & & 7 & 4.4 & 42 & 8.8 & & 13 & 8.2 & 3.3 & 6.9 & & 3 & 1. & 5 & 1.0 & \\
\hline
\end{tabular}

Tabla $4 \mathrm{~B}$

HEMORRAGIA UTERINA ORGANICA

\begin{tabular}{|c|c|c|c|c|c|c|c|c|c|c|c|c|}
\hline \multirow{3}{*}{ Grupo de Edad/Años } & \multicolumn{4}{|c|}{$\begin{array}{l}\text { Polipo } \\
\text { Mioma }\end{array}$} & \multirow{3}{*}{$\begin{array}{l}\text { PS } \\
\mathrm{X} 2 \\
\end{array}$} & \multicolumn{4}{|c|}{$\begin{array}{l}\text { Carcinoma } \\
\text { Endometrial }\end{array}$} & \multicolumn{3}{|c|}{$\begin{array}{l}\text { Hormonas } \\
\text { Exógenas }\end{array}$} \\
\hline & \multicolumn{2}{|c|}{ IMI } & \multicolumn{2}{|c|}{ ISS } & & \multicolumn{2}{|c|}{ IMI } & \multicolumn{2}{|c|}{ ISS } & \multirow{2}{*}{$\begin{array}{l}\text { PS } \\
\mathbf{X} 2\end{array}$} & \multicolumn{2}{|c|}{ IMI } \\
\hline & n & $\%$ & n & $\%$ & & n & $\%$ & n & $\%$ & & n & $\%$ \\
\hline 20-39 & — & & + & & & 1 & 0.6 & 1 & 0.2 & NS & 9 & 1.9 \\
\hline $40-59$ & 2 & 1.2 & 4 & 0.8 & NS & 2 & 1.2 & 3 & 0.6 & NS & 4 & 0.8 \\
\hline Total & 2 & 1.2 & 4 & 0.8 & & 3 & 1.8 & 4 & 0.8 & & 13 & 2.7 \\
\hline
\end{tabular}

El diagnóstico etiológico es confirmatorio por anatomía patológica aceptados universalmente según los criterios de Noyes-Hertig (10), al comparar el comportamiento de la HUD con algunos autores extranjeros y un estudio nacional se encuentran las siguientes cifras Tabla 5.

Tabla 5

\section{COMPORTAMIENTO DE LA HUD SEGUN DIFERENTES} AUTORES

\begin{tabular}{|llll|}
\hline Antor & Ref. & HUD\% & HUO\% \\
\hline Claesen & $(11)$ & 74 & 26 \\
Czernobilsky & $(12)$ & 83 & 17 \\
Cortés & $(13)$ & 76 & 24 \\
Isaacs & $(17)$ & 80 & 20 \\
Presente estudio & & 71 & 29 \\
\hline
\end{tabular}

La hemorragia uterina disfuncional muestra una clara tendencia a que su origen funcional se encuentra en un $70 \%$ y su forma orgánica en un $30 \%$. Es posible que la histeroscopia como ayuda diagnóstica eleve las cifras en favor de la parte orgánica (14).

El comportamiento de la HUD por grupos de edad nos permite inferir que ésta se comporta proporcionalmente en los mismos valores desde los 20 hasta los 60 años. Si bien al aumentar la edad se esperaba un predominio de causa orgánica en este estudio, sólo hay diferencias entre 30-39 años $(\mathrm{P}<0.01)$. Las cifras bajas encontradas por debajo de los 20 años son explicables por el tipo de población que es atendida en estas instituciones $(15,16)$.

Al analizar los tipos de endometrio en la HUD se encuentra que el endometrio secretor disminuye su fre- cuencia con la edad en el IMI - estos datos están de acuerdo a lo informado por la literatura $(4,7,14)$ - en ISS permanece ligeramente elevado $(8.8 \% / 16.2 \%)$ El E. Proliferativo es mayor en el IMI al avanzar la edad frente al ISS $(\mathrm{P}<0.01)$ y lo que nos lleva a plantear la hipótesis que dadas las características socioeconómicas de las dos poblaciones la función endocrina ovárica fuera más prolongada en el ISS que en IMI, dato que sería confirmatorio si la edad de la menopausia estuviera por encima de los 50 años. Por otra parte llama la atención que en el ISS se encuentra un mayor número de endometrios estimulados exógenamente los que nos lleva a pensar que el nivel cultural influye en este tipo de conducta. En un estudio similar en nuestro medio informa E. Secretor $44 \%$ y proliferativo $32 \%$ de un total de 100 biopsias endometriales (13).

La hemorragia uterina orgánica deja unos interrogantes llamativos como la endometritis, la cual si bien pueden ser de origen infeccioso, los estudios sobre el tema no hacen énfasis para descartar tuberculosis como causa secundaria. El embarazo es una entidad que debe estar presente ante una hemorragia en la vida reproductiva y vemos que mantiene cifras de un $3 \%$ en mayores de 40 años. Se debe destacar al tomar la biopsia endometrial en pacientes mayores de 40 años tanto la hiperplasia y $\mathrm{Ca}$. Endometrial y a pesar de las cifras tan bajas $(0,6 \%)$ en una población general encontrar $\mathrm{Ca}$. Endometrial entre los 20-39 años. En un estudio similar sobre un total de 397 pacientes se encuentran valores de $8.3 \%$ para la hiperplasia y en 22.500 biopsias una incidencia de la endometrial (17) de $1.2 \%$ (18). Si comparamos estas cifras con el presente estudio podemos concluir que estas entidades se mantienen dentro de la frecuencia internacional. 


\section{BIBLIOGRAFIA}

1. Shepard BL. The pathology of dysfuncional uterine bleeding. Clin. Obstet. Gynecol. 1984; 11: 227-236.

2. Knobil E. Patterns of hypophysiotrofic signals and gonadotropin secretion in the rhesus monkey. Biol reprod 1981; 24: 44-48.

3. Van Look PFA., Lothian H., Hunter WM. et al. Hipothalamic-pituitaryovarian function in perimenopausal women. Clin. Endocrinology 1977; 7: 13-21.

4. Field Cs. Dysfunctional uterine bleeding. Primary care 1988; 15: 561-574. 5. Spellacy WN. Hemorragias uterinas. Clin. Obstet. Gynecol. 1983; 3: 853867.

6. Fraser IS. The "dysfunctional" uterus: dysmenorrhea and dysfunctional uterine bleeding. In Shearman RP. Clinical Reproductive Endocrinology. Churchill Livingstone 1985; 579-598.

7. Beazley JM. Dysfunctional uterine hemorrhage. BR. J. Med. Hosp. 1972; 7: 573-578.

8. Angel G. La biopsia de endometrio en esterilidad. Rev. Col. Obstet. Ginecol. 1976; 27: 150-152.

9. Sánchez J. Onatra W. Hemorragia uterina disfuncional en el IMI 19871988. Rev. Col. Obstet. Ginecol. 1990; 41: 247-252.

10. Noyes RW., Hertig AT. Dating the endometrial biopsy. Fertil Steril 1950; 1: 3-6.
11. Claessens EA., Cowell CA. Acute adolescent menorrahia. Am. J. Obstet. Gynecol. 1982; 139: 277-279.

12. Czernobilsky B. Utero-ovarian pathology in dysfunctional uterine bleeding. Clin. Obstet. Gynecol. 1970; 13: 416-423.

13. Cortés CC., Serrano GR., Gamboa AG. Hallazgos histológicos en la hemorragia uterina disfuncional. Rev. Col. Obstet. Ginecol. 1983; 34: 169-173.

14. McCausland AM. Hysteroscopic miometrial biopsy: its use in diagnosis adenomyosis and its clinical application. Am. J. Obstet. Gynecol. 1992; 166: 1619-1628.

15. Rybo G., Leman J., Tibbin E. Epidemiology of menstrual blood loss. In baird DT, Michie EA. Mechanism of menstrual bleeding. Raven Press 1985; 181-193.

16. Muran D. Sangrado vaginal en niñas y adolescentes. Clin. Ginecol. Obstet. 1990; 2: 368-386.

17. Isaacs HJ. Diagnosis and management of endometrial disease in reid's controversy in obstetrics and ginecology. Zuspan, Christian Edit. W.B. Saunders Co. Phyladelphya 1983; 601-610.

18. Hofmeiister FJ., Stanhope CR. Diagnosis and management of endometrial disease. In reid's controversy inobstetrics and gynecology. Zuspan, Christian Edit. W.B. Saunders Co. Phyladelphia 1983; 582-599. 